\title{
Multipath Routing protocols in Wireless Sensor Networks: A Survey and Analysis
}

\author{
Mohammad Masdari ${ }^{1}$ and Maryam Tanabi ${ }^{2}$ \\ ${ }^{1}$ Science and Research Branch, Islamic Azad University, West Azarbayjan, \\ Urmia, Iran \\ ${ }^{2}$ Department of Computer Engineering, Science and research Urmia Branch, Islamic \\ Azad University, Urmia, Iran
}

Maryam.Tanabi@gmail.com

\begin{abstract}
Multipath routing protocols improve the load balancing and quality of service in WSN and also provide reliable communication. This paper investigates various multi-path routing protocols of the WSN in the literature and illustrates its benefits. The main elements of these schemes and their classifications based on their attributes have been also discussed. A comparison of these protocols which are of great help to understand the properties and limitations of existing solutions have been done in the following study.
\end{abstract}

Keywords: Sensor network, routing, multipath, Path Discovery

\section{Introduction}

Recent advances in micro-electro-mechanical systems which are highly integrated digital electronics have led to the development of micro-sensors that sensor nodes can sense, measure, and gather information from the environment. These systems can be based on some local decision process and transmit the sensed data to the user that are displayed in Figure 1 [1]. Units of sensory nodes can be listed as a power unit, processing unit, sensing unit, and communication unit. [2]. On the other hand, some potential applications of sensor networks that have been currently pointed out include: military sensing, physical security, traffic surveillance, industrial automation and environment monitoring [3]. Since, data transmission from the target area towards the sink node is the main task of wireless sensor networks, the utilized method to forward data packets between each pair of source-sink nodes is an important issue that should be addressed in developing these networks [5]. Routing in sensor networks is very challenging issue which is due to several characteristics that distinguish them from other contemporary communication and wireless ad hoc networks. First of all, it is not possible to build a global addressing scheme for the deployment of sheer number of sensor nodes. Therefore, classical IP-based protocols cannot be applied to sensor networks. Second, in contrary to typical communication networks, almost all applications of sensor networks require the flow of sensed data from multiple regions (sources) to a particular sink. Third, since multiple sensors may generate the same data within the vicinity of a phenomenon generated data traffic has significant redundancy in it. Fourth, sensor nodes are tightly constrained in terms of transmission power, on-board energy, processing capacity and storage, and thus require careful resource management [4]. According to the aforementioned differences between WSN and traditional wireless networks, numerous routing protocols 
were proposed over the past decade to address the routing challenges imposed by the new features of WSN of which multipath routing is one of them.

In this paper, Section 2 analyzes the benefits and elements of Multipath routing protocol in WSN, Section 3 displays a Taxonomy of Multipath Routing Protocols in WSN and Section 4 presents the concluding remarks.

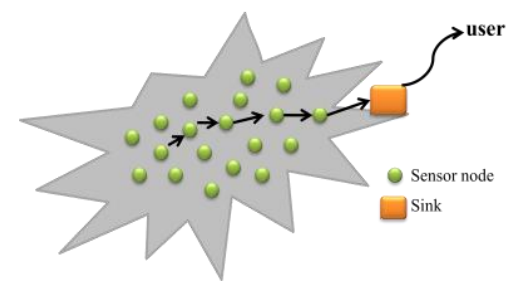

Figure 1. Scheme of Wireless Sensor Networks

\section{Multipath Routing Protocol in WSN}

The multipath routing technique which has demonstrated its efficiency to improve wireless sensor performance is efficiently used to find alternate paths between sources and sink. This approach is considered as one of the existing solutions to cope with the limitations of routing [5]. In this section, the benefits and elements of multipath routing will be explained.

\subsection{Benefits of Multipath Routing}

Here, the researcher describes how these benefits are achieved.

- Reliability and Fault-Tolerance: The original idea behind using multipath routing approach in WSN was to provide path resilience (against node or link failures) and reliable data transmission. In the fault tolerance domain, whenever a sensor node cannot forward its data packets towards the sink, it can benefit from the availability of alternative paths to salvage its data packets from node or link failures [7].

- Load Balancing: As traffic distribution is not equal in all links in the network, spreading the traffic along multiple routes can alleviate congestion in some links and bottlenecks [9].

- QoS Improvement: QoS support in terms of network throughput, end-to-end latency and data delivery ratio is an important objective in designing multipath routing protocols for different types of networks [7].

- Reduced Delay: The delay is minimized in multipath routing because backup routes are identified during route discovery [8].

- Bandwidth Aggregation: Splitting data to the same destination into multiple streams while everyone is routed through a different path, the effective bandwidth can be aggregated. This strategy is particularly beneficial when a node has multiple low bandwidth links but it requires a bandwidth that is greater than the one which an individual link can provide [8].

\subsection{Elements of a Multipath Routing Protocol}

Three elements of multipath routing include path discovery, traffic distribution, and path maintenance which are explained below. 


\subsubsection{Path Discovery}

Since data transmission in wireless sensor networks is commonly performed through multi-hop data forwarding techniques, the main task of the route discovery process is to determine a set of intermediate nodes that should be selected to construct several paths from the source nodes towards the sink node [6].

- Disjoint Multipath Routing: In sensor-disjoint path routing, the primary path is available whereas the alternate paths are less desirable as they have longer latency. The disjoint makes those alternate paths independent of the primary path. Thus, if a failure occurs on the primary path, it remains local and does not affect any of those alternate paths [11].

- Braided Multipath Routing: To construct the braided multipath, first the primary path is computed. Then, for each node on the primary path, the best path is computed while it does not include that node. Those best alternate paths are not necessarily disjoint from the primary path and are called idealized braided multi-paths [11].

\subsubsection{Traffic Distribution}

In spite of path discovery of multiple paths issue, another important issue that is discussed here is traffic distribution Multipath routing algorithms that optimally split traffic into a given set of paths investigated in the context of flow control .It is worth noting that the selection of the routing paths is another major design consideration that has a drastic effect on the resulting performance [10].

- Number of Paths: A protocol can use a single path and keep the rest as backups, or it can utilize multiple paths in a round-robin fashion, with only one path sending at a time [8].

- Allocation Granularity: The traffic allocation strategy that is used deals with how the data is distributed amongst the paths. The choice of allocation granularity, which is important in traffic allocation, specifies the smallest unit of information allocated to each path [12].

\subsubsection{Path Maintenance}

In multipath routing, the process of route discovery can be done when one of the routes fails or it may occur after all of the routes fail. Waiting for all routes to fail before performing a route discovery would result in a delay before new routes are available. It should be noted that initiating the process of route discovery can make high overheads whenever one of the routes fails [12].

\section{Taxonomy of Multipath Routing Protocols}

Figure 2 introduces a comprehensive classification of the multipath routing protocols proposed for wireless sensor networks. This taxonomy classifies the existing multipath routing protocols into four main categories and we analysis them in the next subsections. 


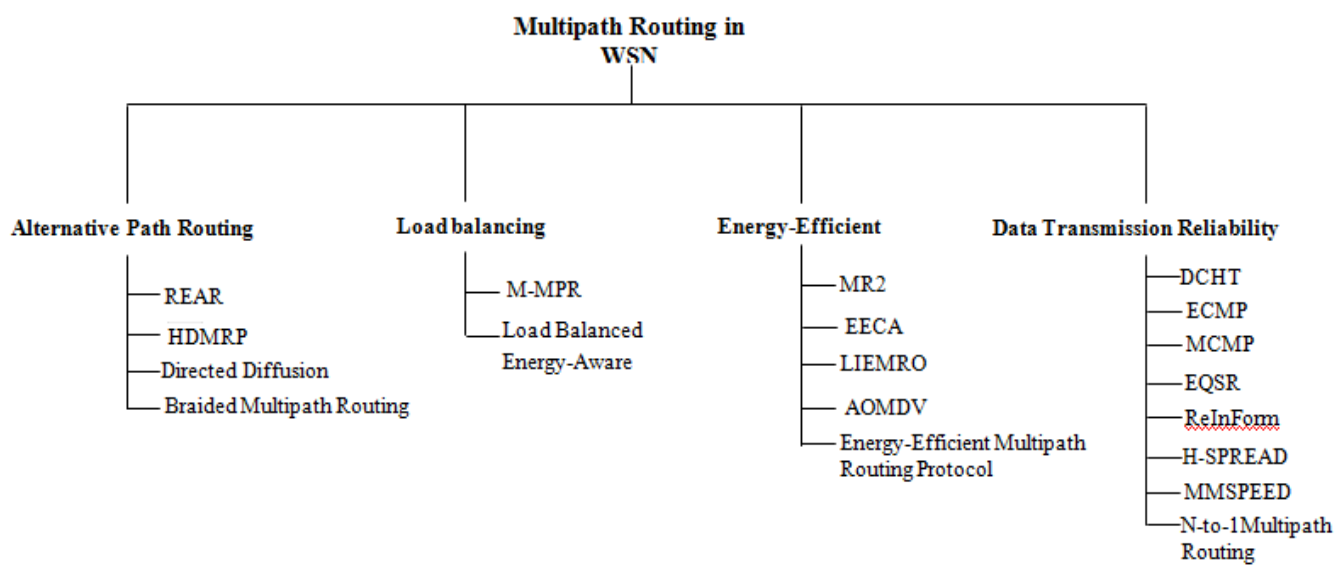

Figure 2. Taxonomy of the Existing Multipath Routing Protocols

\subsection{Alternative Path Routing}

One of the reasons behind developing multipath routing is to provide route failure protection, and increase resiliency to route failures. Discovering and maintaining multiple paths between the source destination pair improves the routing performance by providing alternative routes. When the primary path fails, an alternative path will be used to transfer the data while the multiple paths are not used simultaneously [14]. Alternate Path Routing can be used to provide a Fault-Tolerance routing and to reduce the frequency of routing in the process of rediscovery. Table 1, shows the number of the protocols of this category.

\subsubsection{Directed Diffusion}

Directed diffusion provides mechanisms for a sink node to flood a query toward the sensors of interest as well as for intermediate nodes to set up gradients to send data along the routes toward the sink node. A key feature of directed diffusion is that every sensor node can be aware, which means that nodes store and interpret interest packets, rather than merely forwarding them along the route [13].

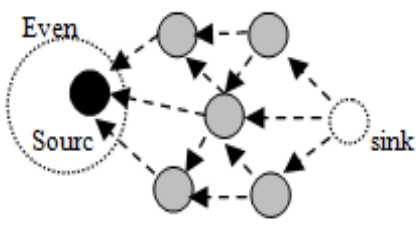

(a)

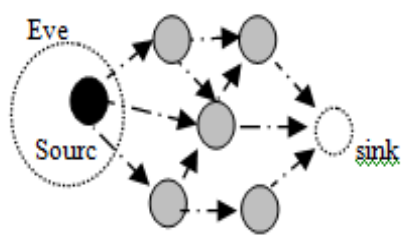

(b)

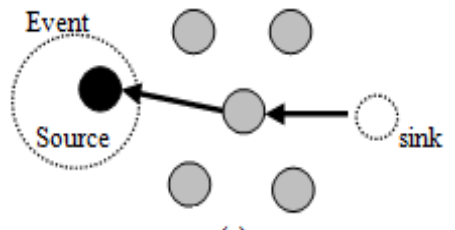

(c)

Figure 3. (a) Interest Propagation; (b) Gradient Setup; (c) Path Reinforcement and Data Transmission

\subsubsection{Braided Multipath Routing Protocol}

A desirable goal of multipath routing is to deliver data along the paths. If failure of paths happens, in order to recover without flooding the network to rediscover, it is helpful to construct a small number of alternative paths called braided multipath on the existed paths called main paths. For each node on the main path, the construction mechanism of such 
alternative paths is to find the best path from the source to the destination that does not contain that node [17].

\subsubsection{REAR: Reliable Energy Aware Routing Protocol}

Reliable Energy Aware Routing (REAR) protocol considers residual energy capacity of each sensor node in establishing routing paths and supports multi-path routing protocol for reliable data transmission. Furthermore, REAR allows each sensor node to confirm the success of data transmission to other sensor nodes by supporting the DATA-ACK oriented packet transmission [15].

\subsubsection{HDMRP: An Efficient Fault-Tolerant Multipath Routing}

In HDMRP, sink neighbors are called root nodes and root neighbors are called sub-roots. The protocol uses Route Request (RREQ) message propagation through nodes to construct multiple energy-node-disjoint paths between each node and the sink. Each non root node maintains a routing table containing an entry for each discovered path. During paths construction, a node may receive several RREQ messages corresponding to one or several paths. Instead of using all nodes as reducing elements belonging to only one path, HDMRP introduces a controlled intersection at master nodes by allowing them to forward several RREQ messages to their neighbors [16].

\subsection{Data Transmission Reliability}

Sending multiple copies of data across different paths, multiple paths can be used simultaneously to improve reliability. As long as one of the multiple paths does not fail, the receiver node will receive the data [14]. To increase the probability of data delivery, the redundancy of data is delivered in the form of multiple copies of the same packet which travels to the destination among multiple paths [18].

\subsubsection{H-SPREAD}

H-SPREAD proposed an extension to find more extra routes at cost of additional messages, by breaking the property of using "one message per node". When a sensor node discovers a new alternative path, it informs its neighborhood about it. Recursively, this information is propagated through the network to maximize the number of disjoint paths per node [20].In fact, in less challenging situations, the improvement would be more significant. Correspondingly, the reliability performance shows that H-SPREAD is able to maintain pretty good message delivery ratio in the face of both link and node failures that the situations we studied are very stressful [23].

\subsubsection{N-to-1 Multipath Routing Protocol}

This protocol finds different node-disjoint paths between a sink and a source node and also multi-paths, which are used to distribute the traffic to improve the reliability and security of the data transmission by traversing the tree. However, as illustrated in Figure 4, the N-to-1 routing protocol does not take into account the node energy level during the route construction phase [21]. 

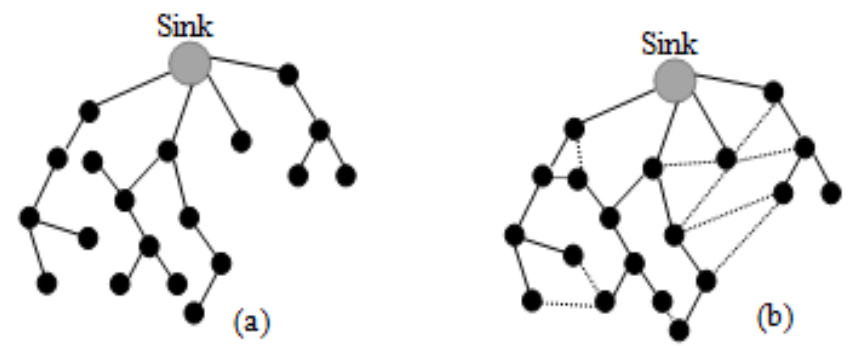

Figure 4. N-to-1 Multipath Routing (a) Spanning Tree Created by Flooding (b) A Simple Multipath Extension of Flooding

\subsubsection{Multipath Multispeed Protocol (MMSPEED)}

MMSPEED (Multi path Multi SPEED) is an extension of the SPEED protocol [25]. It is characterized by offering multi-speed transmission and the establishment of more than one path to the destination. Indeed, for each offered speed, a QoS level and an additional path can be set to improve the quality of traffic. This protocol allows to send packets with respect to end delay parameter required by the applications in order to avoid congestion and reduce the packet loss rate [22].It is designed to provide probabilistic QoS differentiation with respect to timeliness and reliability domains. For the timely delivery of packets, MMSPEED provides multiple delivery speed options for each incoming packet. Thus, the protocol is scalable and adaptable to large networks. The only limitation of the protocol is that the energy metric is not taken into consideration [27].

\subsubsection{Multi-Constrained QoS Multipath Routing (MCMP)}

Multi-constrained QoS multi-path routing (MCMP) protocol that uses braided routes to deliver packets to the sink node according to certain QoS requirements expressed in terms of reliability and delay. The protocol's objective is to utilize the multiple paths to augment network performance with moderate energy cost. However, the protocol always routes the information over the path that includes minimum number of hops to satisfy the required QoS, which leads in some cases to more energy consumption [14].This MCMP routing algorithm trades precise link information for sustainable computation, memory and overhead for resource limited sensor nodes [32].

\subsubsection{Energy Constrained Multipath Routing (ECMP)}

Energy constrained multi-path routing (ECMP) extends the MCMP protocol by formulating the QoS routing problem as an energy optimization problem constrained by reliability, play-back delay, and geo-spatial path selection constraints. This protocol trades between minimum number of hops and minimum energy by selecting the path that satisfies the QoS requirements and minimizes energy consumption [14].

\subsubsection{Delay-Constrained High-Throughput Protocol for Multipath Transmission (DCHT)}

This protocol is applied for multipath video streaming over wireless sensor networks. Multiple disjoint paths can achieve high throughput and desirable delay and meet the QoS requirement of multimedia streaming [33]. 


\subsubsection{Energy-Efficient and QoS-based Multipath Routing Protocol (EQSR)}

An energy efficient and QoS aware multipath routing protocol namely EQSR that maximizes the network lifetime through balancing energy consumption across multiple nodes, uses the concept of service differentiation to allow high important traffic to reach the sink node within an acceptable delay, reduces the end to end delay through spreading out the traffic across multiple paths, and increases the throughput through introducing data redundancy. EQSR uses the residual energy, node available buffer size, and Signal-to-Noise Ratio (SNR) to predict the best next hop through the paths construction phase [24]. On the other hand, EQGR considers reliability, timeliness and energy to select next optimum neighbor node for data forwarding. To forward reliable data, the authors consider multipath forwarding with optimum link quality [36].

\subsubsection{Reliable Information Forwarding (ReInForm) Using Multiple Paths in Sensor Networks}

In ReInForM scheme, multiple copies of the same packet are transmitted over randomly chosen routes. Specifically, it is assumed that a packet is destined to a sink node and that each node knows its hop distance to the sink as well as the hop distances of all its immediate neighbors. Packet duplication can occur not only at the source node but also at every intermediate node. An intermediate node has to decide two things: the number of copies to create and the upstream nodes to which the packet is actually forwarded. With respect to the latter choice, ReInForM prefers nodes which are really closer to the sink, otherwise the choice is random. This distributes the load over many nodes and avoids quick depletion of nodes along a "good" route [19].

\subsection{Load Balancing}

The main goal of load balancing is to use the available network resources in order to minimize the risk of traffic congestion. When a link becomes over-utilized and causes congestion, multipath routing protocols can be chosen to divert traffic through alternate paths to ease the burden of the congested link. Load balancing can be achieved by spreading the traffic along multiple routes which alleviates congestion and bottlenecks [32].

\subsubsection{Meshed Multipath Routing (M-MPR)}

M-MPR achieves better load distribution and requires less route maintenance overhead with respect to packet forwarding along a preferred route. Evaluation of MMPR in terms of throughput shows that it outperforms its node equivalent as well as the receiver complexity equivalent D-MPR. Besides, the throughput gain of M-MPR is more appropriate for longer end-to-end distance for comparable throughput performance. It distributes traffic more evenly along the meshed route and requires less route maintenance overhead compared to packet forwarding along the primary route [26].

\subsubsection{Load Balanced, Energy-Aware Communications}

This scheme utilizes a multi-path routing scheme with energy-aware and load balanced route selection based on the poll-reply communication model. While poll messages flood, multiple paths are constructed from each sensor back to the base station. These paths are all of equal minimal hop distance to the base station. Upon receiving a poll request, a sensor node reports that it has collected through one of the paths. The path selection can be either random or based on the energy usage at neighbors. The multipath routing builds a mesh 
structure for data reply, which reduces the congestion and improves the reliability of data delivery [29].

\subsection{Energy-Efficient}

One of the goals of the energy aware routing (EAR) protocols is to select the best path so that the total energy consumed by the network is minimized. A serious drawback of the minimum energy routing is that nodes will have a wide difference in energy consumption. Nodes on the minimum energy paths will quickly drain out while the other nodes remain intact. This will result in the early death of some nodes. Another objective of the EAR is to maximize the system lifetime, which is defined as the duration when the system starts to work till any node runs out of energy, or till a certain number of nodes run out of energy, or till the network is partitioned, etc., [ 28].

\subsubsection{Energy-Efficient Multipath Routing Protocol}

This protocol is a distributed, scalable, and localized multipath search protocol to discover multiple node-disjoint paths between the sink and source nodes and also a load balancing algorithm to distribute the traffic over the multiple paths discovered [30]. Since the path construction algorithm of AODVM depends on overhearing neighboring nodes' transmissions, the following approach is based on AOMDV. It is also worth noting that permanent overhearing requires to Keep the receiver constantly in the receive state, which is contrary to the scope of the energy-efficient MAC [31].

\subsubsection{Maximally Radio-Disjoint Multipath Routing (MR2)}

The main objective of this routing is to provide necessary bandwidth to multimedia applications through non interfering (radio disjoint) paths while increasing the network lifetime. To achieve two fold goals, an incremental approach should be adopted where only one path is built at once for a given session. Additional paths are built when required, typically in case of path congestion or lack of bandwidth. When a given path is selected to be used, all nodes interfering with it are put in a passive state. Passive nodes do not further take part in the routing process so they could not be used to form a new path that consequently will not interfere with previously built ones. Moreover, passive nodes can be put in sleep or idle modes, thus allowing for energy saving and hence increasing the network lifetime [35].

\subsubsection{Energy-Efficient and Collision-Aware Multipath Routing Protocol (EECA)}

An energy efficient and collision aware (EECA) node disjoint multipath routing algorithm for WSNs is presented in [34]. The main idea of EECA is to use the broadcast nature of wireless communication to avoid collisions between two discovered routes without extra overhead. Additionally, this protocol restricts the route discovery flooding and adjusts node transmit power with the aid of node position information, resulting in energy efficiency and good performance of communication that proposed scheme in terms of the average packet delivery ratio, the average end-to-end delay, the average residual energy, and the number of nodes alive [38].

\subsubsection{Low-Interference Energy-Efficient Multipath Routing Protocol (LIEMRO)}

This protocol is mainly designed to improve packet delivery ratio, lifetime, and latency, through discovering multiple interference-minimized node disjoint paths between source node and sink node. In addition, LIEMRO includes a load balancing algorithm to distribute source 
node's traffic over multiple paths based on the relative quality of each path. It consists of a multipath routing protocol and a load balancing algorithm. In this approach, a set of nodedisjoint interference-minimized paths are established from the source to the sink, while these paths impose minimum interference over each other (to minimize route coupling effect). Moreover, extra routes are only established if they don't decrease data reception rate at the sink node [37].

\subsubsection{Energy Efficient Adaptive Multipath Routing}

In this protocol, the continuous use of the minimum energy path deprives the nodes energy quickly and it takes time to determine an alternate path increase. Multipath routing schemes distribute traffic among the sink, while they impose minimum interference over each other (to minimize route coupling effect). Moreover, extra routes are only established if they don't decrease data reception rate at the sink node [37].

Table 1presents the comparison of various multipath routing protocols in WSN.

Table 1. Comparative Comparison of Multipath Routing in WSN

\begin{tabular}{|c|c|c|c|c|c|c|c|}
\hline Protocols & $\begin{array}{c}\text { Path } \\
\text { Disjointedness }\end{array}$ & $\begin{array}{l}\text { Energy- } \\
\text { Efficient } \\
\end{array}$ & Delay & $\begin{array}{c}\text { Fault- } \\
\text { Tolerance }\end{array}$ & Reliability & $\begin{array}{c}\text { scalable } \\
\text { large }\end{array}$ & QoS \\
\hline H-SPREAD & Node-disjoint & No & No & No & Yes & No & No \\
\hline N-to-1 & Node-disjoint & No & No & No & Yes & No & No \\
\hline MMSPEED & Partially disjoint & No & Yes & No & Yes & Yes & Yes \\
\hline MCMP & Partiallydisjoint & No & Yes & No & Yes & Yes & Yes \\
\hline ECMP & Partially disjoint & Yes & Yes & No & Yes & No & Yes \\
\hline DCHT & Node-disjoint & No & Yes & No & Yes & No & Yes \\
\hline EQSR & Node-disjoint & Yes & Yes & Yes & No & No & Yes \\
\hline ReInForm & Link-disjoint & No & No & No & Yes & No & No \\
\hline M-MPR & Node-disjoint & No & No & No & No & No & No \\
\hline $\begin{array}{l}\text { Load Balanced, } \\
\text { Energy-Aware } \\
\text { Communications }\end{array}$ & Partially disjoint & Yes & No & No & Yes & No & No \\
\hline REAR & Node-disjoint & Yes & No & No & Yes & No & No \\
\hline $\begin{array}{c}\text { Directed } \\
\text { Diffusion }\end{array}$ & Node-disjoint & Yes & No & Yes & No & No & No \\
\hline HDMRP & Node-disjoint & No & No & Yes & No & No & No \\
\hline
\end{tabular}

\section{Conclusions}

Multipath routing is one of the effective methods to improve the capacity of network and productivity of sources under heavy traffic conditions. This paper presented a comprehensive analysis of multipath routing protocols in wireless sensor networks. The researchers also specified the challenges related to designing multipath routing protocols in WSN and compared various properties of these routing protocols. The mentioned comparison is of great importance to understand the existing solutions and also design new multipath routing protocols.

\section{References}

[1] J. Yick, B. Mukherjee and D. Ghosal, "Wireless sensor network survey”, (2008), pp. 2292-2330.

[2] M. Augusto M. Vieira and D. C. da Silva Junior, "Survey on Wireless Sensor Network Devices".

[3] C.-Y. Chong and S. P. Kumar, "Sensor Networks: Evolution, Opportunities, and Challenges", (2003), vol. 91. 
[4] K. Akkaya and M. Younis, “A survey on routing protocols for wireless sensor networks”, (2005), pp. 325349.

[5] Y. Chen and N. Nasser, "Energy-Balancing Multipath Routing Protocol for Wireless Sensor Networks", (2006).

[6] M. T. Tarique, K. E. Adibi and S. Erfani, "Survey of Multipath Routing Protocols for Mobile Ad Hoc Networks", J. Netw. Comput. (2009), pp. 1125-1143.

[7] R. Marjan, D. Behnam, A. B. Kamalrulnizam and M. Lee, "Multipath Routing in Wireless Sensor Networks: Survey and Research Challenges", (2012).

[8] J. Tsai and T. Moors, "A Review of Multipath Routing Protocols: From Wireless Ad Hoc to Mesh Networks".

[9] C. Neves Fonseca, "Multipath Routing for Wireless Mesh Networks", (2010).

[10] R. Banner and A. Orda, "Multipath Routing Algorithms for Congestion Minimization", IEEE/ACM transactions on networking, (2007).

[11] S. Kumar Singh, M. P. Singh and D. K. Singh, "Routing Protocols in Wireless Sensor Networks-A Survey", International Journal of Computer Science \& Engineering Survey (IJCSES), (2010).

[12] C. Intanagonwiwat, R. Govindan and D. Estrin, "Directed Diffusion: A Scalable and Robust Communication Paradigm for Sensor Networks", Proceedings of the 6th Annual International Conference on Mobile Computing and Networking (MobiCom '00), Boston, MA, (2000), pp. 56-67.

[13] M. Chen, T. Kwon and Y. Choi, "Energy-efficient differentiated directed diffusion (EDDD) in wireless sensor networks, (2005), pp. 1-15.

[14] J. Ben-Othman and B. Yahya, "Energy efficient and QoS based routing protocol for wireless sensor networks", (2010).

[15] K.-Y. Shin, J. Song, J. W. Kim, M. Yu and P. Soo Mah, "REAR: Reliable Energy Aware Routing Protocol for Wireless Sensor Networks", (2007).

[16] A. Hadjidj, A. Bouabdallah and Y. Challal, "HDMRP: An Efficient Fault-Tolerant Multipath Routing Protocol for Heterogeneous Wireless Sensor Networks", Social Informatics and Telecommunications Engineering, (2011), pp. 469-482.

[17] Y. Yang, H. Zhong, Y. Sun and I. Yang, "Network coding based reliable disjoint and braided multipath routing for sensor networks", Journal of Network and Computer Applications, (2010), pp. 422-432.

[18] B. Deb, S. Bhatnagar and B. Nath, "ReInForM: Reliable Information Forwarding using Multiple Paths in Sensor Networks".

[19] A. Willig and H. Karl, "Data Transport Reliability in Wireless Sensor Networks-A Survey of Issues and Solutions".

[20] Y. Challala, A. Ouadjaoutb, N. Laslab, M. Bagaab and A. Hadjidja, "Secure and efficient disjoint multipath construction for fault tolerant routing in wireless sensor networks", Journal of Network and Computer Applications, (2011), pp. 1-41.

[21] B. Yahya and J. Ben-Othman, "REER: Robust and Energy Efficient Multipath Routing Protocol for Wireless Sensor Networks".

[22] W. Ben Jaballah and N. Tabbane, "Multi path Multi SPEED Contention Window Adapter", IJCSNS International Journal of Computer Science and Network Security, (2009).

[23] W. Lou and Y. K, "H-SPREAD: a Hybrid Multipath Scheme for Secure and Reliable Data Collection in Wireless Sensor", IEEE, (2006), pp. 1320-1330.

[24] A. RanjideRezaie and M. Mirnia, "CMQ: Clustering based Multipath routing algorithm to improving QoS in wireless sensor networks", IJCSI International Journal of Computer Science Issues, (2012).

[25] E. Felemban and C.-G. Lee, "MMSPEED: Multipath Multi-SPEED Protocol for QoS Guarantee of Reliability and Timeliness in Wireless Sensor Networks", 738 IEEE transactions on mobile computing, (2006), vol. 5.

[26] E. Felemban and C.-G. Lee, "MMSPEED: Multipath Multi-SPEED Protocol for QoS Guarantee of Reliability and Timeliness in Wireless Sensor Networks", 738 IEEE transactions on mobile computing, (2006), vol. 5.

[27] R. Sumathi and M. G. Srinivas, "A Survey of QoS Based Routing Protocols for Wireless Sensor Networks", J Inf Process Syst, (2012).

[28] W. Lou, W. Liu and Y. Zhang, "Performance Optimization using Multipath Routing in Mobile Ad Hoc and Wireless Sensor Networks", (2005).

[29] X. Hong, M. Gerla and H. Wang, "Load Balanced, Energy-Aware Communications for Mars Sensor Networks", (2002).

[30] Y. Ming Lu and V. W. S. Wong, “An energy-efficient multipath routing protocol for wireless sensor networks", International journal of communication systems, (2007), pp. 747-766.

[31] P. Hurni and T. Braun, "Energy-Efficient Multi-Path Routing in Wireless Sensor Networks". 
[32] A. Jayashree, G. S. Biradar and V. D. Mytri, "Review of Multipath Routing Protocols in Wireless Multimedia Sensor Network -A Survey", International Journal of Scientific \& Engineering Research, (2012).

[33] S. Li, R. Neelisetti, C. Liu and A. Lim, "Delay-Constrained High Throughput Protocol for Multi-Path Transmission over Wireless Multimedia Sensor Networks".

[34] Z. Wang, E. Bulut and B. K. Szymanski, "Energy Efficient Collision Aware Multipath Routing for Wireless Sensor Networks", (2008).

[35] M. Maimour, "Maximally Radio-Disjoint Multipath Routing for Wireless Multimedia Sensor Networks", (2008)

[36] A. Ghaffari, N. Firuz and H. RahbariBannaeian, "REMP: Reliable and Energy Balancing Multi-Path Routing Algorithm for Wireless Sensor Networks", World Applied Sciences Journal, (2011), pp. 737-744.

[37] M. Radi, B. Dezfouli, S. Abd Razak and Kamalrulnizam Abu Bakar, "LIEMRO: A Low-Interference Energy-Efficient Multipath Routing Protocol for Improving QoS in Event-Based Wireless Sensor Networks", International Conference on Sensor Technologies and Applications, (2010).

[38] A. Premita and M. Katiyar, "A Review on Power Efficient Energy- Aware Routing Protocol for Wireless Sensor Networks", International Journal of Engineering Research \& Technology (IJERT), (2012). 
International Journal of Future Generation Communication and Networking Vol.6, No.6 (2013) 